\title{
Germination des Graines et Croissance en Pépinière et en Champ des Plantules de Detarium senegalense au Bénin
}

\author{
Benjamin A. K. Dossa, \\ Bienvenue Sourou, \\ Christine Ouinsavi,
}

Laboratoire d'Etudes et de Recherches Forestières (LERF), Faculté d'Agronomie, Université de Parakou, Bénin

Doi:10.19044/esj.2020.v16n12p38～URL:http://dx.doi.org/10.19044/esj.2020.v16n12p38

\section{Résumé}

La plantation ou le semis est une méthode certaine pour asseoir rapidement sur le sol une couverture végétale de protection. L'utilisation réussie des semences ne peut être examinée sans une compréhension parfaite de leur physiologie. La présente étude sur Detarium senegalense a pour objectif de déterminer les paramètres de germination et la croissance des plants en pépinière et en champ. Les graines utilisées pour la germination ont subi plusieurs traitements. Le suivi de la croissance des plantules en pépinière et d'autres mis en champ a été effectué. Les données des paramètres de germination et de croissance ont été collectées et analysées. Les résultats ont montré que les graines de Detarium senegalense ont une forte potentialité de germination sans traitement avec un taux de germination de $76 \%$ et un temps de latence de 20 jours. Les résultats du test de Kruskal-Wallis et d'analyse de la variance (ANOVA) ont montré que la durée de trempage des graines dans l'eau froide à température ambiante a un effet négatif sur le taux de germination des graines. En ce qui concerne le suivi de la croissance des plants, les résultats ont montré que les plants de Detarium senegalense ont un accroissement assez rapide. Les plants en pépinière ont atteint en moyenne une hauteur de $83,8 \pm 2,28 \mathrm{~cm}$ après huit mois en pépinière. Tandis que les plants en champs quant à eux ont atteint une hauteur moyenne de 137,33 7,08 $\mathrm{cm}$. Les plants en champ ont une vigueur de croissance assez élevée que les plants en pépinière. Detarium senegalense a l'aptitude d'être utilisée dans les programmes de reboisement et de restauration des forêts dégradées après quatre mois en pépinière.

Mots clés : Detarium Senegalense, Dormance Des Graines, Croissance Juvénile, Essai En Champ 


\title{
Germination of Seeds and Growth in the Nursery and Field of Seedlings of Detarium senegalense in Bénin
}

\author{
Benjamin A. K. Dossa, \\ Bienvenue Sourou, \\ Christine Ouinsavi,
}

Laboratoire d'Etudes et de Recherches Forestières (LERF),

Faculté d'Agronomie, Université de Parakou, Bénin

\begin{abstract}
Planting or sowing is a sure method for quickly placing a protective plant cover on the ground. The successful use of seeds cannot be examined without a perfect understanding of their physiology. The present study on Detarium senegalense aims to determine the parameters of germination and growth of plants in the nursery and in the field. Different treatments have been carried out on the seeds used for germination. The growth of seedlings in the nursery and other field crops was monitored. Data on germination and growth parameters were collected and analyzed. The results showed that the seeds of Detarium senegalense have a strong potential for germination without treatment with a germination rate of $76 \%$ and a standby time of 20 days. The results of the Kruskal-Wallis test and analysis of variance (ANOVA) showed that the duration of soaking the seeds in cold water at room temperature has a negative effect on the germination rate of the seeds. With regard to monitoring the growth of the plants, the results showed that the plants of Detarium senegalense have a fairly rapid growth. Nursery plants reached an average height of $83.8 \pm 2.28 \mathrm{~cm}$ after eight months in the nursery. While the field plants have reached an average height of $137.33 \pm 7.08 \mathrm{~cm}$. Plants in the field have a fairly high growth vigor than plants in the nursery. Detarium senegalense has the ability to be used in reforestation and restoration programs for degraded forests after four months in the nursery.
\end{abstract}

Keywords: Detarium Senegalense, Seed Dormancy, Juvenile Growth, Field Trial

\section{Introduction}

Les formations végétales sont soumises aux effets conjugués des changements climatiques et des activités humaines entrainant une dégradation de plus en plus croissante des écosystèmes. Ainsi des programmes de 
développement se sont focalisés sur les plantations d'espèces végétales à croissance rapide, d'intérêts socio-économiques et qui notamment présentent divers avantages pour le développement rural ont été vulgarisés (Hamawa et al., 2020).

L'exploitation incontrôlée des formations végétales entraine la régression rapide, voire la disparition totale de certaines espèces très importantes pour la population (Yossi et al., 2006). Il est alors très utile de reconstituer les peuplements naturels non seulement à cause des rôles écologiques et environnementaux qu'ils jouent dans la vie de l'Homme, mais aussi pour satisfaire la demande croissante des populations en produits forestiers divers.

Detarium senegalense est une espèce fortement exploitée pour son bois qui offre de nombreux usages comme bois d'œuvre et de feu, mais aussi pour les produits alimentaires, médicinaux, artisanaux et dans la cosmétique. Les amandes des graines de D. senegalense et les fruits ont fait par le passé, l'objet d'un commerce florissant en Afrique de l'Ouest, notamment de divers pays en direction du Nigeria et du Ghana (Neuenshwander et al. 2011, Diop et al., 2010, Diop, 2013, Sabaly K. 2014 ; Dangbo et al., 2019). Pour alimenter ce commerce au Bénin, les forêts classées des Monts Kouffé, Bantè, Idadjo, Bassila et Tchaourou constituaient les pôles majeurs de collecte des fruits de Detarium senegalense (Neuenshwander et al. 2011). Cette collecte et vente d'amandes ont largement impacté négativement la régénération naturelle de l'espèce et conséquemment sa survie. Detarium senegalense a un statut d'espèce vulnérable sur la liste rouge de l'UICN du Bénin (Neuenschwander et al. 2011). Selon ces mêmes auteurs, les forêts classées qui abritent en grande partie l'espèce sont toutes détruites et n'existent en réalité plus que sur papier dans la majorité des cas. Par ailleurs, les effets des différentes pressions exercées sur cette espèce sont aggravés par la variabilité climatique et par le fait que sa sylviculture n'est pas maîtrisée pour permettre la reconstitution des peuplements dégradés et le développement des plantations. La densité des individus adultes de même que celle de la catégorie des régénérations est très faible (Dossa et al., 2019). Mais depuis maintenant plusieurs années, la collecte et la vente des amandes de D. senegalense au Bénin ont totalement régressé, voire interrompu (Dossa et al., 2019). Les mêmes auteurs indiquent qu'en dépit de l'arrêt de la collecte, la régénération naturelle est demeurée rare. Les jeunes semis et gaulis sont presque inexistants et ceci, malgré la présence relativement importante des fruits et graines sous les semenciers de quelques populations naturelles reliques de D. senegalense des forêts galeries. Plusieurs questions se dégagent de tous ces constats : qu'est ce qui explique l'absence de régénération naturelle sous les pieds mère malgré la présence des graines au sol après la fructification? Qu'est ce qui explique aussi la non survie des plantules issues de la germination par endroit des graines. La non germination 
des graines et la non survie des plantules seraient elles liées à l'habitat propice de l'espèce ? La réponse à ces questions nécessite la mise en place d'un essai de germination des graines de Detarium senegalense et le suivi de quelques plants issus de germination en champ et en pépinière. Etant donné que la bonne connaissance des techniques de multiplication ou de régénération des espèces ligneuses constitue un préalable indispensable pour le suivi durable et la restauration de la végétation, il est alors indispensable de connaître les mécanismes de la régénération naturelle de Detarium senegalense qui devrait orienter sa sylviculture et conduire à sa restauration et domestication. C'est pourquoi la présente étude a été initiée dans le but d'élucider les mécanismes de multiplications appropriées pour la production de plants en vue de l'intégration de l'espèce dans les programmes de reboisement futurs. Les objectifs visés par cette étude sont (i) d'identifier les facteurs à l'origine de la non germination des graines d'une part, et d'autre part, de rechercher les facteurs d'amélioration de la germination des graines de Detarium senegalense et (ii) d'évaluer la croissance juvénile de l'espèce en pépinière et en champ.

\section{Matériel et méthodes}

\subsection{Site d'expérimentation}

Le test de germination des graines de Detarium senegalense a été réalisé dans la ferme de la Faculté d'Agronomie de l'Université de Parakou au Bénin situé à $9^{\circ} 21^{\prime}$ de latitude Nord, à $2^{\circ} 36^{\prime}$ de longitude Est à une altitude moyenne de $350 \mathrm{~m}$ et présente un relief assez modeste. C'est un milieu dont le climat est de type tropical humide (climat Sud soudanien) caractérisé par l'alternance d'une saison de pluies (Mai à Octobre) et d'une saison sèche (Novembre à Avril). C'est en Décembre-Janvier que l'on enregistre les températures les plus basses à Parakou. La précipitation moyenne annuelle est de $1200 \mathrm{~mm}$ dont le maximum survient entre juillet, août et septembre. L'essai de germination a été conduit d'avril 2018 à juin 2019.

\subsection{Matériel végétal}

- $\quad$ Description de la graine de D. senegalense

Les travaux ont porté sur les graines et les fruits de Detarium senegalense. Le fruit de cette espèce a une forme globuleuse à ovoïde de 4 à $6 \mathrm{~cm}$ de diamètre et est lisse, verdâtre, devenant à terme noirâtre, à pulpe verdâtre et très fibreuse. Il dispose d'un noyau atteignant $4,5 \mathrm{~cm}$ de diamètre et contient une seule graine de forme aplatie et souvent plus ou moins ronde. Ce fruit est constitué de trois parties principales (Diop, 2013):

- l'épicarpe vert foncé, dur pour les fruits immatures, vert clair tirant sur le marron et cassant pour les fruits mûrs ; 
- le mésocarpe verdâtre (pulpe) entremêlé de fibres insérées sur le noyau correspondant à la portion comestible du fruit, elle représente environ $45 \%$ de sa masse ;

- le noyau volumineux, ligneux recouvert de mailles fibreuses renfermant une seule graine ovoïde et aplatie de couleur marron foncé, il représente également $45 \%$ de la masse du fruit. A l'intérieur de la graine se trouve l'amende de forme aplatie et oléagineuse qui est beaucoup plus commercialisé.

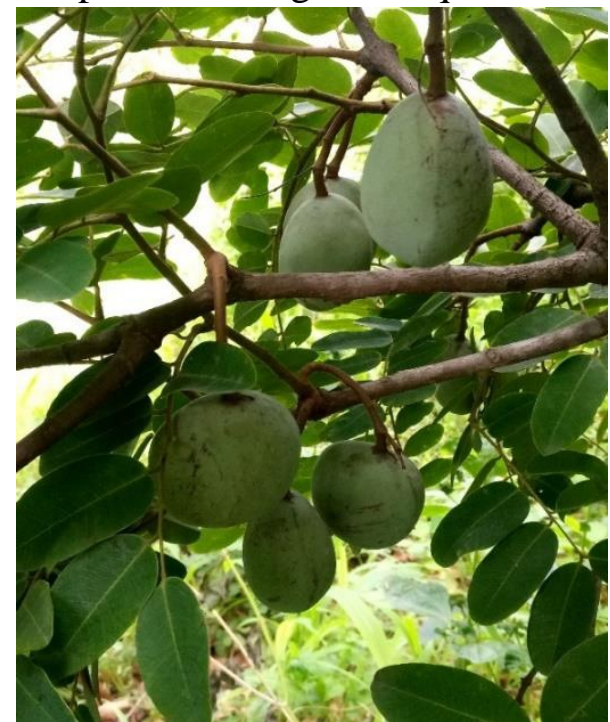

Photo 1 : Fruits de Detarium senegalense

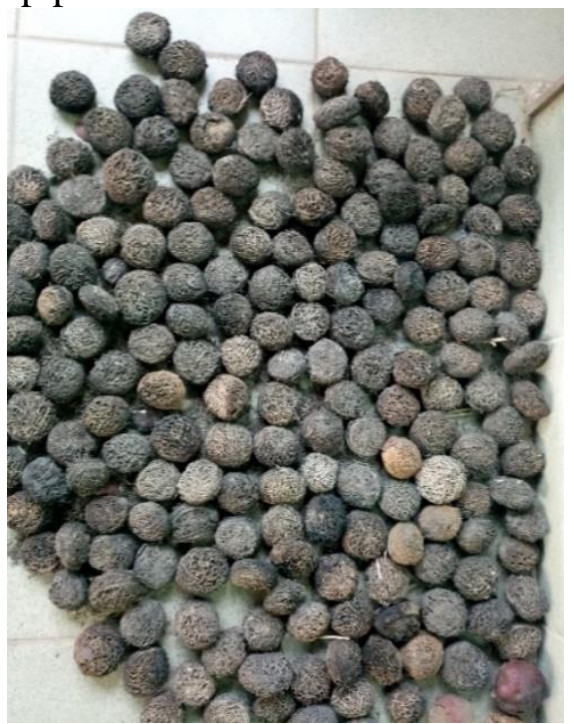

Photo 2: Graines de Detarium senegalense Source : Dossa, mai 2018

\subsection{Approche méthodologique}

\subsubsection{Collecte des données}

\section{- $\quad$ Provenance des graines de Detarium senegalense}

Les graines ayant servi au test de germination ont été fraichement récoltées en mars 2018 sur des individus semenciers sains et vigoureux dans les forêts denses et les forêts galeries des forêts classées de Bassila et de Pénéssoulou et dans quelques terroirs villageois riverains (Photo 3). Les caractéristiques géomorphologiques et physionomiques des zones de provenance des graines sont proches de celles du milieu de l'essai de germination et de transplantation des plants produits. En effet les forêts classées de Bassila et de Pénéssoulou se retrouvent dans la zone soudano-guinéenne au Bénin et soumises à un régime pluviométrique unimodal. La pluviométrie moyenne annuelle est de $1200 \mathrm{~mm}$ de pluie réparties d'avril à octobre. 


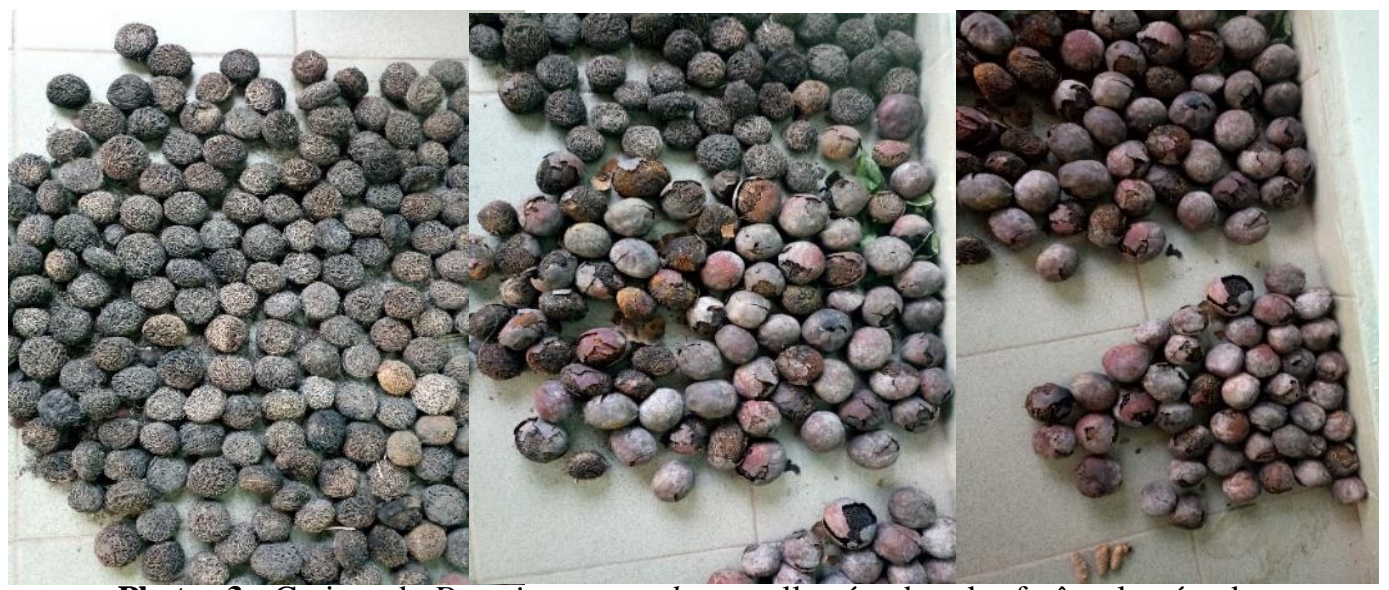

Photos 3 : Graines de Detarium senegalense collectées dans les forêts classées de Pénéssoulou et de Bassila

- Germination graines de Detarium senegalense

Compte tenu de la dureté du péricarpe des graines de Detarium senegalense, les semences ont été soumises aux traitements pré-germinatifs suivants :

$\checkmark$ Témoins sans traitements

$\checkmark$ Trempage dans l'eau froide pendant 24 heures

$\checkmark$ Trempage dans l'eau froide pendant 48 heures

Pour chaque test, 50 graines ont été prélevées du stock et mises en germination dans 50 sachets polyéthylènes biodégradables avec comme substrat le sol forestier. Au total 150 graines ont été donc utilisées pour l'essai de germination. Les graines ont été semées à une profondeur de 2 à $5 \mathrm{~cm}$ selon la grosseur des graines. Les comptages de graines germées ont été effectués tous les jours pendant une période de trente (30) jours comptant à partir de la première graine germée. L'essai a été conduit dans le mois d'avril pendant lequel les pots ont été arrosés deux fois par jour, matin et soir. L'essai a été conduit sous l'ombrière pour résoudre le problème de l'ensoillement. Une graine est dite germée si la tigelle pointe à la surface du sol. Ces différentes données collectées ont permis de calculer les paramètres suivants :

$>$ Le taux de germination: (Nombre de graines germées/Nombre de graines mises à germer) $\times 100$;

$>$ La durée de germination (en jours) : durée entre la première et la dernière germination ;

$>$ Le délai de germination ou temps de latence (en jours) : durée entre le semis et la première germination

$>$ Le taux de croissance des plantules de Detarium senegalense en pépinière et en champs. 


\section{- Croissance des plantules de Detarium senegalense}

Pour suivre la croissance des plants en pépinière, trente (30) plants les plus vigoureux ont été sélectionnées et des mesures ont été prises au niveau de chaque plantule. Tous les deux (2) mois, les paramètres suivants ont été mesurés : la hauteur des plants mesurés du collet au bourgeon terminal à l'aide d'une règle graduée en centimètre, le diamètre au collet au moyen d'un pied à coulisse gradué en millimètre, le nombre de feuilles et de ramification par comptage simple. De même, quinze (15) plants ont été également mis en champ pour le suivi de la croissance. Les paramètres mesurés au niveau des plants en pépinière ont été les mêmes paramètres mesurés au niveau des plants en champs. La plantation en champ a été faite après trois (3) mois en pépinière avec des plantules disposant en moyenne de cinq feuilles. Aussi bien les plantules en pépinière que les plantations en champs ont été arrosées deux fois par jour, matin et soir pendant les huit (8) mois d'essai. Cet effectif par essai a été choisi compte tenu des plantules ayant atteint une taille acceptable pour leur suivi en pépinière et en champ.

\subsubsection{Analyse des données}

Le test de Kruskal-Wallis et l'analyse de variance (ANOVA) ont été utilisés pour évaluer l'effet des traitements sur le taux de croissance et comparer les moyennes des différents facteurs étudiés sur les plants en pépinière et en champ. Les analyses ont été faites avec le logiciel R3.2.4.

En ce qui concerne l'essai de suivi de la croissance, les taux d'accroissement relatif (Relative Growth Rate $=$ RGR) en hauteur $(h)$, en diamètre au collet $(D c)$ et le nombre de feuilles $(n f)$ ont été calculés à partir des formules ci-après (Castel \& Terradas, 1994) :

$\mathrm{RGR}_{h}=[\ln (h \mathrm{~T} 2)-\ln (h \mathrm{~T} 1)] /(\mathrm{T} 2-\mathrm{T} 1)$ pour la croissance en hauteur ;

$\mathrm{RGR}_{D c}=[\ln (D c \mathrm{~T} 2)-\ln (D c \mathrm{~T} 1)] /(\mathrm{T} 2-\mathrm{T} 1)$ pour la croissance du diamètre au collet ;

$\mathrm{RGR}_{n f}=[\ln (n f \mathrm{~T} 2)-\ln (n f \mathrm{~T} 1)] /(\mathrm{T} 2-\mathrm{T} 1)$ pour exprimer le nombre de feuilles. Avec $h$ la hauteur exprimée en centimètre, $D c$ le diamètre au collet, $n f$ le nombre de feuilles et $\mathrm{T}$ le temps en un intervalle de deux mois.

\section{Résultats}

\subsection{Paramètres de germination des graines de Detarium senegalense}

Le tableau I présente les paramètres de germination des graines de Detarium senegalense. Le temps de latence est de vingt (20) jours quel que soit le traitement pré germinatif appliqué aux graines de Detarium senegalense. Quant à la durée de germination, elle est de 21 jours chez les graines non trempées, 18 jours chez les graines trempées pendant 24 heures et de 16 jours chez les graines trempées pendant 48 heures. Le taux de germination est de $38 \%$ pour les graines trempées à l'eau pendant $24 \mathrm{~h}, 26 \%$ 
pour celles trempées à l'eau pendant $48 \mathrm{~h}$ contre $76 \%$ pour le lot témoin (graines non trempées). Cette variation observée est statistiquement significative $(\mathrm{SCE}=1565,6, \mathrm{~F}=10,68, \mathrm{ddl}=3, \mathrm{P}<0,0001)$.

La figure 1 présente l'évolution du taux de germination des graines.

Tableau I : Paramètres de germination des graines de Detarium senegalense

\begin{tabular}{llll}
\hline Paramètres de germination des graines & \multicolumn{2}{l}{} \\
\hline Traitements & Temps de latence & Durée de germination & Taux de \\
& (jours) & (jours) & germination (\%) \\
Témoins & 20 & 21 & 76 \\
Trempage 24 h & 20 & 18 & 38 \\
Trempage 48 h & 20 & 16 & 26 \\
\hline
\end{tabular}

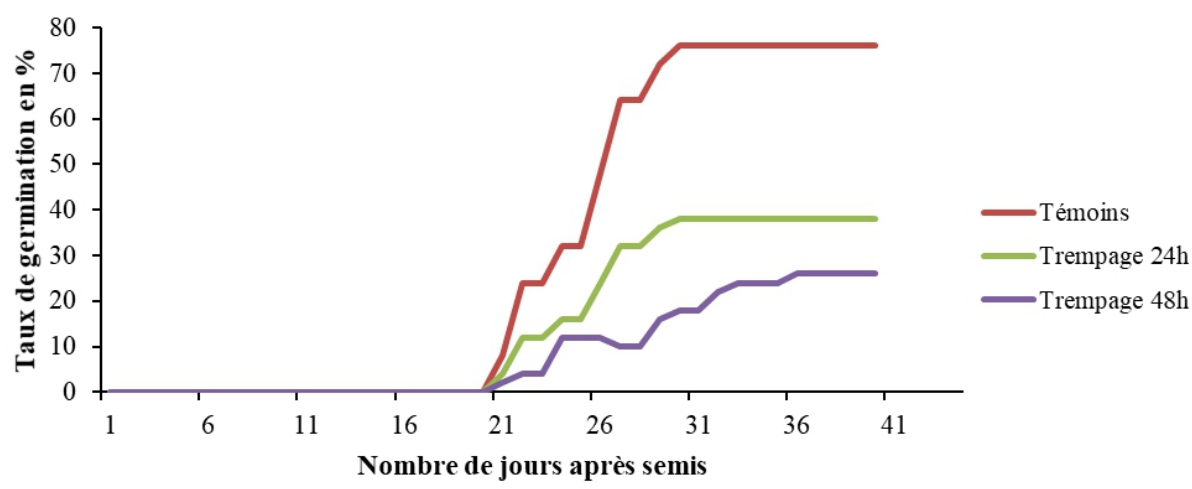

Figure 1 : Evolution du taux de germination des graines de Detarium senegalense

\subsection{Croissance des plantules de Detarium senegalense en pépinière}

Les plants de Detarium senegalesnse ont une croissance très rapide. La hauteur moyenne des plants a atteint $45 \mathrm{~cm}$ en pépinière après trois mois. Pour la croissance du diamètre au collet, elle est de $0,5 \mathrm{~cm}$ en moyenne. De l'apparition de la plantule jusqu'à la fin de la durée de germination, la hauteur moyenne des plantules est de $20,23 \mathrm{~cm}$ et le diamètre moyen au collet est de $0,51 \mathrm{~cm}$ avec un nombre moyen de feuilles de 3,12. La figure 2 ( $\mathrm{a}$ et $\mathrm{b}$ ) présente l'évolution de la croissance en hauteur et en diamètre des plantules de Detarium senegalense. Dans l'intervalle de huit mois d'essai, la moyenne des hauteurs des plantules est passée de $20,23 \mathrm{~cm}$ à $83,8 \mathrm{~cm}$. Quant au diamètre au collet, la moyenne est passée de $0,51 \mathrm{~cm}$ à $1,18 \mathrm{~cm}$. S'agissant du nombre moyen de feuilles dans cet intervalle de temps, il est passé de 3 à 12. Le taux d'accroissement relatif en hauteur de ces plants en pépinière est de 1,92 pour $\mathrm{T} 0 ; 2,31$ pour $\mathrm{T} 1 ; 2,15$ pour $\mathrm{T} 2$ et 2,34 pour $\mathrm{T} 3$ ( $\mathrm{T}$ représentant les périodes bimensuelles consécutives, Figure 3). Le taux d'accroissement relatif en diamètre au collet est de $-0,1$ pour $\mathrm{T} 0 ; 0,39$ pour $\mathrm{T} 1 ; 0,1$ pour $\mathrm{T} 2$ et 0,13 pour T3 (T représentant les périodes bimensuelles consécutives, Figure 4). 

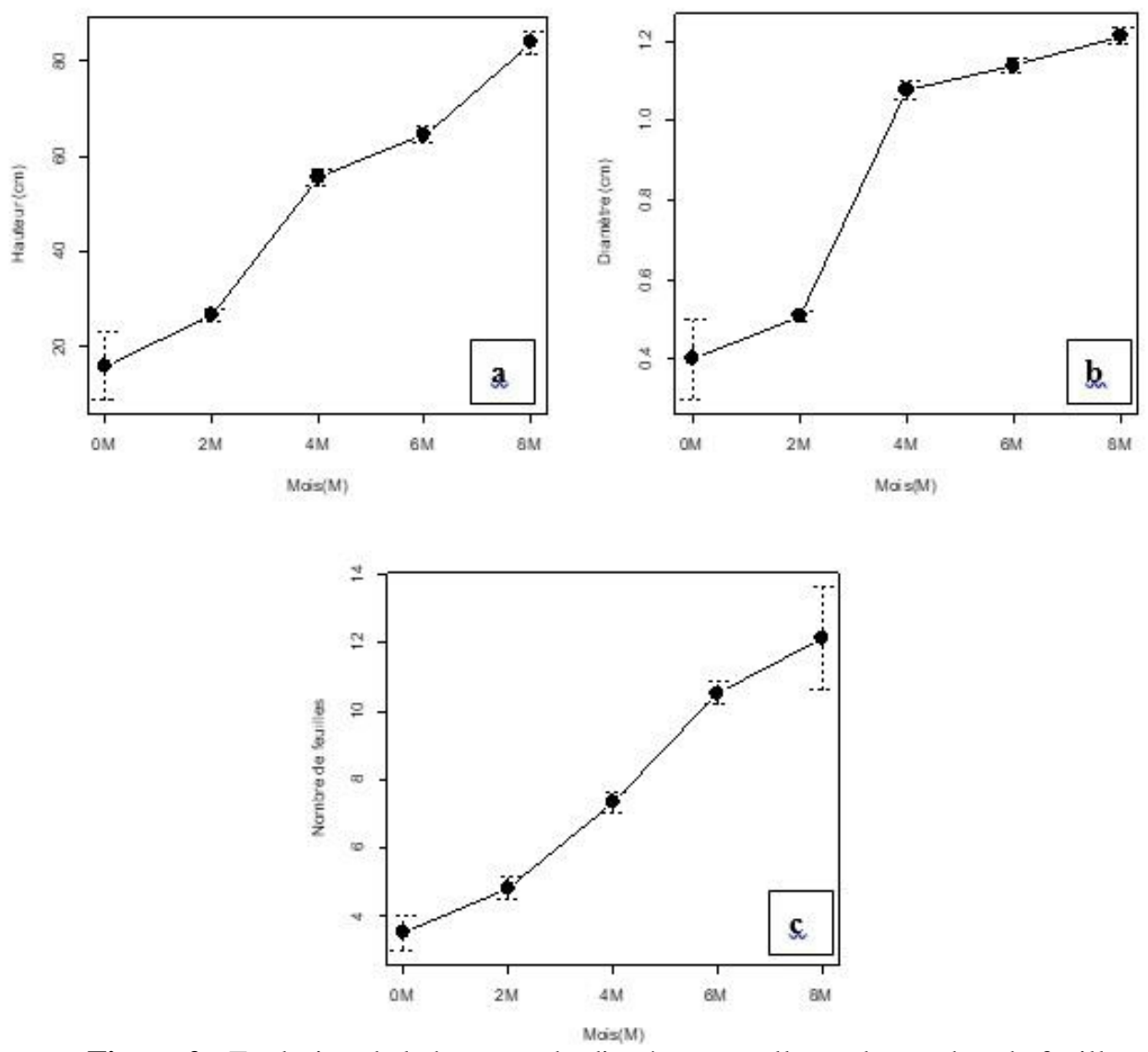

Figure 2 : Evolution de la hauteur, du diamètre au collet et du nombre de feuilles des plants de Detarium senegalense en pépinière

La croissance de Detarium senegalense est assez considérable aussi bien en hauteur et en diamètre. On note une corrélation entre différents paramètres de croissance des plantules de Detarium senegalense. Plus l'espèce croit en hauteur, plus elle produit un nombre important de feuilles et de ramifications (Figure 5, photo 4). 

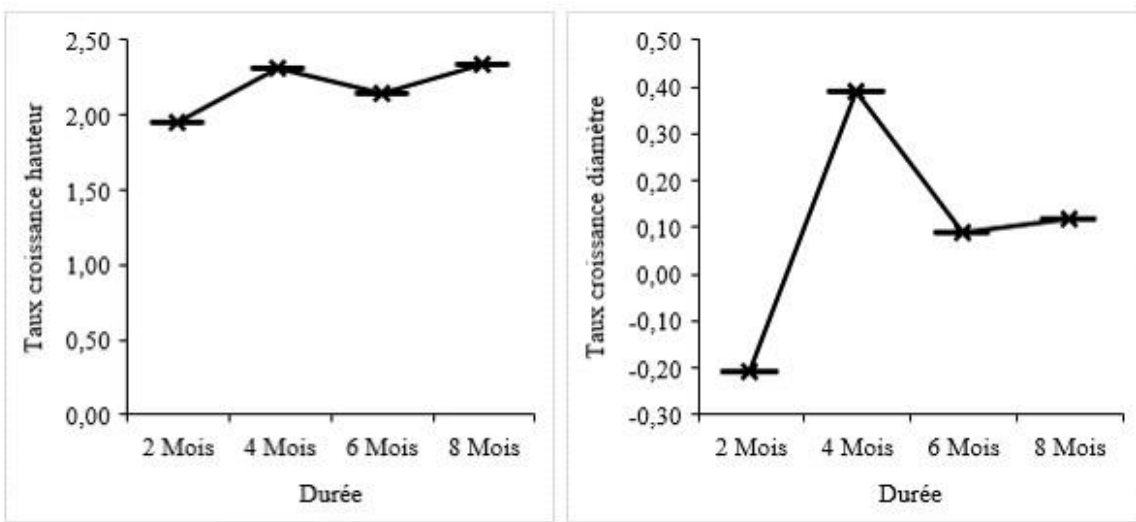

Figure 3 : Évolution du taux de croissance en Figure 4 : Evolution du taux de croissance des hauteur des plantules de $D$. senegalense en pépinière plantules en diamètre de $D$. senegalense en pépinière

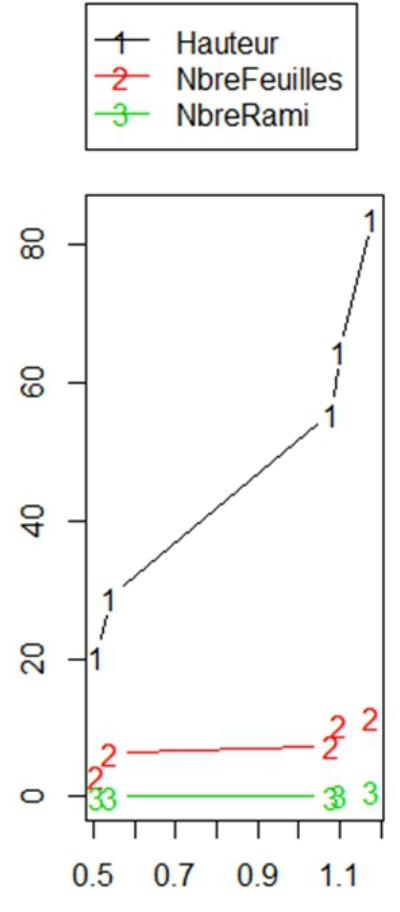

DiamCollet

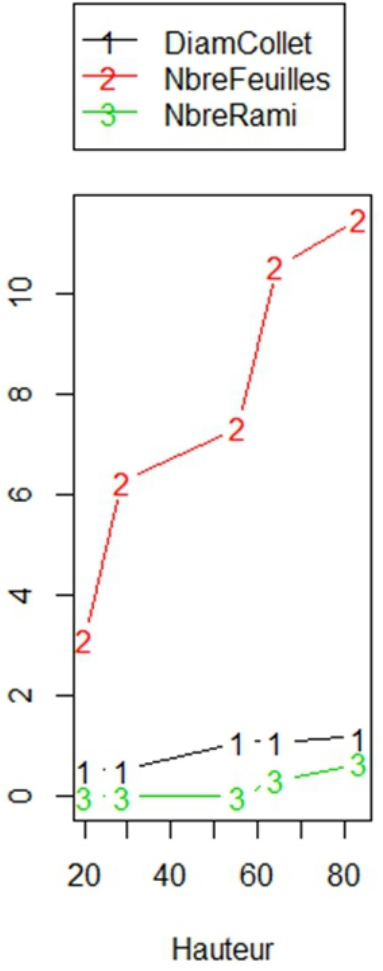

Hauteur

Figure 5 : Corrélation entre les différents paramètres de croissance des plantules de Detarium senegalense en pépinière

\subsection{Croissance des plantules de Detarium senegalense en champ}

Les plants de Detarium senegalense en champ ont une croissance plus rapide comparativement à ceux en pépinière. A la date de mise en terre des plants, la moyenne des paramètres mesurés était de $49,6 \mathrm{~cm}$ pour la hauteur ; $0,72 \mathrm{~cm}$ pour le diamètre au collet et 8 pour le nombre de feuilles. Huit mois 
après la plantation en champ des plants, la moyenne des différents paramètres mesurés est de $137,33 \mathrm{~cm}$ pour la hauteur ; 1,95 pour le diamètre au collet et 57,5 pour le nombre de feuilles (Figure 6). Le taux d'accroissement relatif en hauteur de ces plants en champ est 2,35 pour T0 ; 2,4 pour T1 ; 2,47 pour T2 et de 2,53 pour T3 (T représentant les périodes bimensuelles consécutives, Figure 7) et celui du diamètre au collet est de 0,34 pour $\mathrm{T} 0 ; 0,28$ pour $\mathrm{T} 1$; 0,42 pour $\mathrm{T} 2$ et 0,36 pour $\mathrm{T} 3$ ( $\mathrm{T}$ représentant les périodes bimensuelles consécutives, Figure 8).
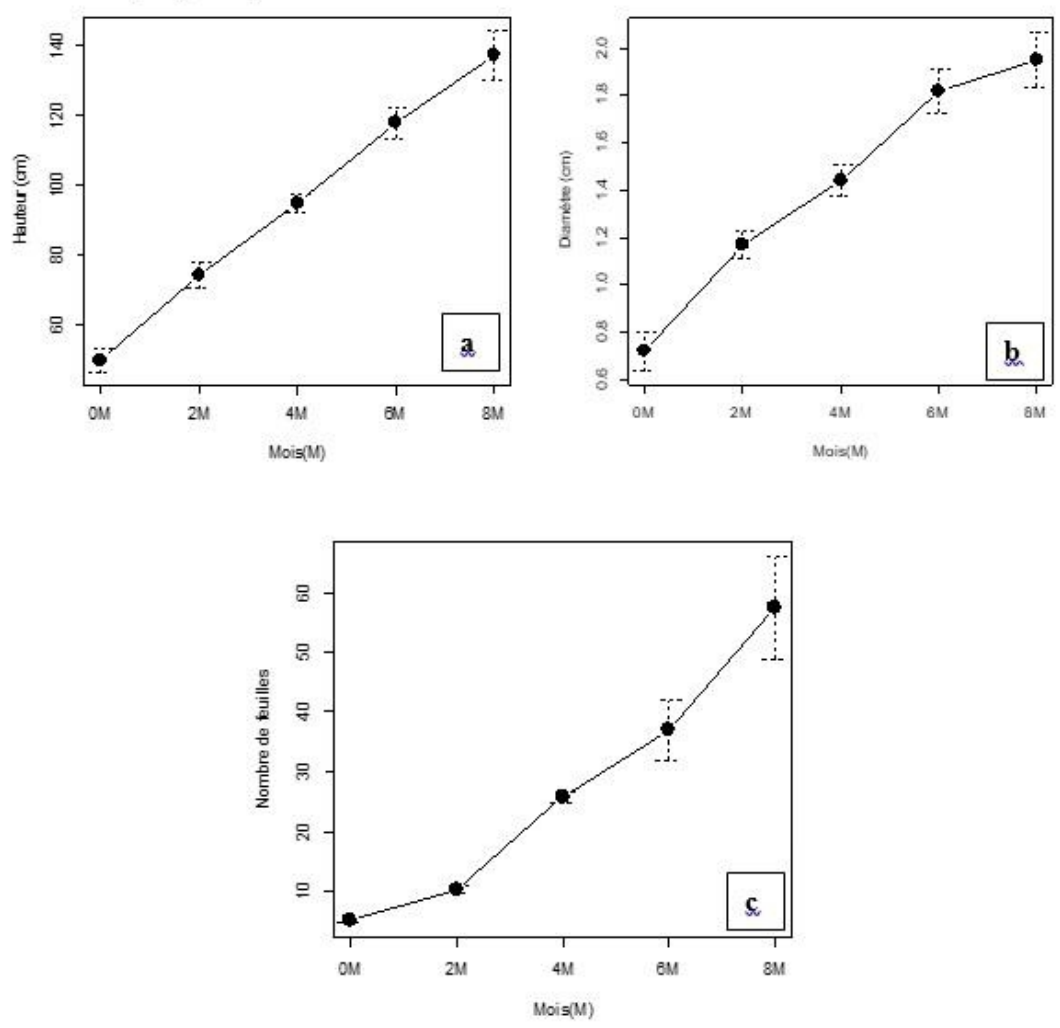

Figure 6 : Evolution de la hauteur, du diamètre au collet et du nombre de feuilles des plants de Detarium senegalense en champ 


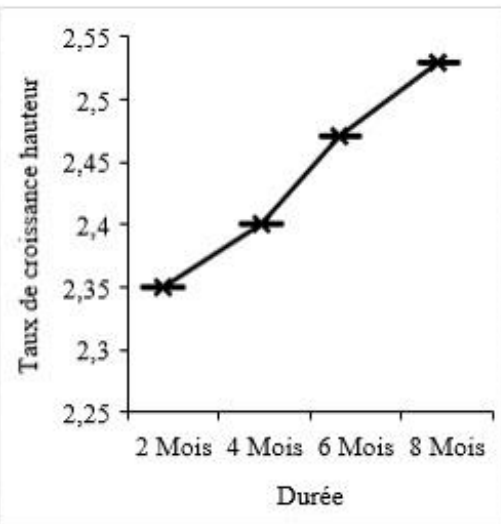

Figure 7 : Évolution du taux de croissance en hauteur des plantules de D. senegalense en champ

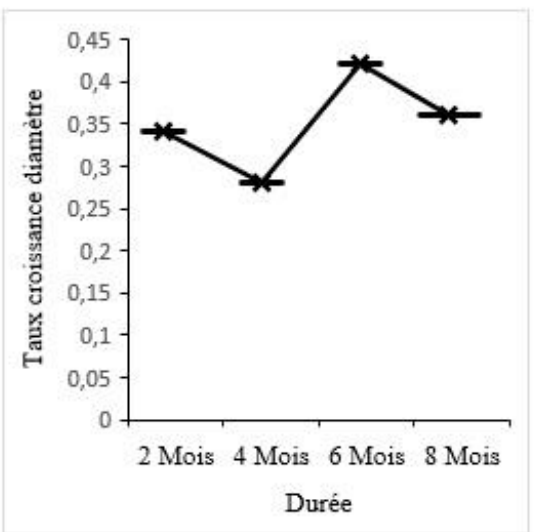

Figure 8 : Evolution du taux de croissance en diamètre des plantules de D. senegalense en champ
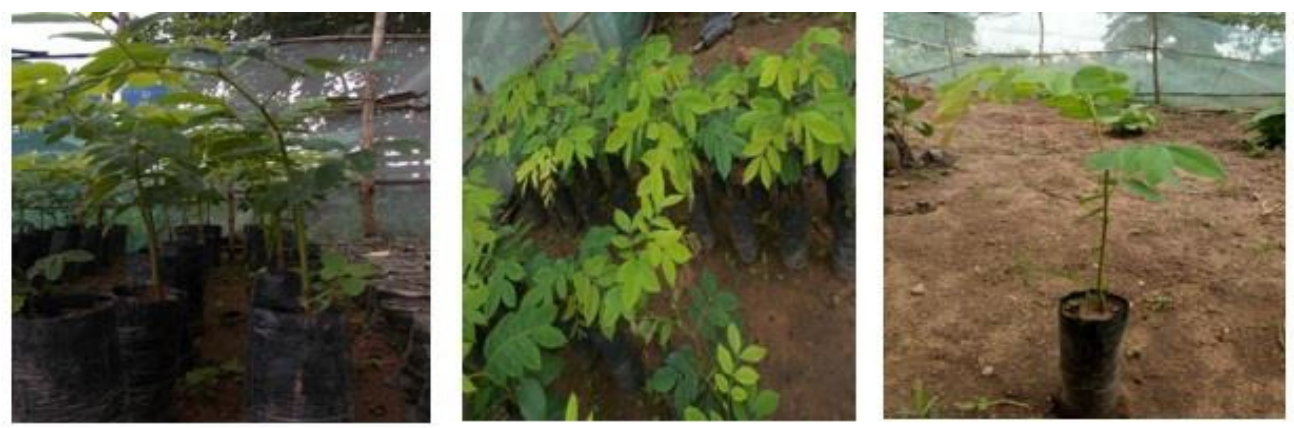

Photos 4 : Plants de Detarium senegalense en pépinière

\section{Discussion}

Les travaux de germination réalisés sur Detarium senegalense ont montré que le taux de germination est relativement acceptable $(76 \%)$ par rapport à d'autres espèces dont les graines sont également oléagineuses; il s'agit de Disopyros mespiliformis avec un taux de germination de 55,4 \% (Ado et al., 2017), de Scerocarya birrea avec un taux de germination de 68,33\% (Haminou et al., 2013). Les résultats ont montré que les témoins ont un taux de germination plus élevé que les graines trempées dans l'eau froide. Le trempage des graines de Detarium senegalense n'a pas amélioré le temps de latence et a par contre un effet significativement négatif sur le taux de germination des graines. Les graines de Detarium senegalense fraîchement récoltées et semées ont une forte aptitude à germer. Les graines semées ont germé au bout de vingt (20) jours contrairement à la littérature qui indique que les graines de Detarium senegalense germent au bout de 6 à 10 semaines après semis (https://uses.plantnet-project.org/fr/Detarium_senegalense_(PROTA). 
Ceci peut être lié aux caractéristiques géomorphologiques et physionomiques des zones de provenance des graines qui sont proches de celles du milieu de l'essai de germination et de transplantation des plants produits. Cela peut également être lié à la non conservation des graines utilisées pour la germination. Detarium senegalense peut donc germer facilement par graine sans aucun traitement de ces graines. Tandis que selon les travaux de Diop (2013), les graines de D. senegalense trempées dans l'eau froide pendant 24 heures donnent un bon taux de germination.

S'agissant du suivi de la croissance des juvéniles, cette étude a permis d'observer que les plants de Detarium senegalense ont une croissance assez rapide. Le suivi des paramètres de croissance et surtout la croissance en hauteur a montré que les plants de Detarium senegalense ont un fort potentiel de croissance en pépinière et plus en champ. Cette potentialité de croissance lui permet d'obtenir des plants adéquats pour la plantation et son exploitation en bois de service et bois d'œuvre. Le taux de croissance des plants de cette espèce, surtout les plants en pépinière est à la fois évolutif et régressif. Ceci pourrait être justifié par l'alternance des saisons qui s'observe au cour de l'année. Pendant les saisons pluvieuses, nous observons un taux d'accroissement beaucoup plus important. S'agissant du taux de croissance au niveau des plants en champ, une tendance à la fois évolutive et régressive des différents paramètres mesurés est constatée. Ces mêmes alternances des saisons qui agissent sur la croissance des plantules ont été abordé dans les travaux de Amani et al., (2015) et d'Ado et al., (2017). Les saisons pluvieuses sont favorables à la croissance des plants de Detarium senegalense. C'est une espèce qui tolère les zones humides. Les résultats de cette étude ont montré que Detarium senegalense n'a aucun problème de germination et de croissance des plantules. L'absence et la non survie des régénérations seraient dues aux habitats propices de l'espèce qui rend difficile la germination des graines et la survie des plants. Comme facteurs empêchant la régénération de l'espèce, nous noterions le non contact des graines au sol dans les forêts denses, la tombée des graines dans les points d'eaux le long des cours d'eaux dans les forêts galeries. La disparition de certaines plantules de l'espèce ayant régénéré dans le lit des cours d'eaux en forêt galerie est due au transport par le courant des cours d'eaux en saison des pluies. Tandis que dans les forêts denses, la non survie de ces plantules pourrait être liée aux ombrages des pieds mères qui empêchent à ces plantules de bénéficier des rayons solaires pour accroitre et survivre. Les difficultés de germination et de survie rencontrées par les plantules de D. senegalense ont été évoquées dans les travaux de Dossa et al., (2019). 


\section{Conclusion}

Cette présente étude qui a porté sur la germination et la croissance des plants de $D$. senegalense, une espèce fruitière à importance économique et en voie de disparition au Bénin a permis d'affirmer que la multiplication par graine de cette espèce est très facile en pépinière avec un taux de germination supérieur à $70 \%$ avec les graines non trempées. Les plantules issues de la germination ont une croissance plus rapide. Ce bon taux de germination des graines et de croissance rapide des plantules a permis d'obtenir des plantules plus vigoureuses qui peuvent être transplantées en milieu naturel ou en plantation après peu de temps (moins de quatre mois) passé en pépinière. La croissance des plantules évolue en fonction des saisons. Pendant les saisons pluvieuses, elles ont une croissance plus rapide qu'en saison sèche. Les différents paramètres mesurés au niveau de l'ensemble des plants mis en champ sont plus évolutifs que ceux en pépinière. La seule façon d'améliorer le taux de germination pour les prochaines études est de faire une bonne sélection des graines à germer. D. senegalense peut donc être produit en pépinière et utilisé dans les programmes de plantation et de reboisement des espaces dégradés en produisant en pépinière les jeunes plants juste après la collecte des graines sous les semenciers en milieu naturel.

\section{References:}

1. Ado A., Issoufou H. B.A., Dan G. I., Toudou D. A. K., Mahamane A., Saadou M. (2017). Effet de prétraitements, de substrats et de stress hydriques sur la germination et la croissance initiale de Diospyros mespiliformis Hochst. Ex A.DC. European Scientific Journal, edition Vol.13, No.21 ISSN: 1857 - 7881 (Print) e - ISSN 1857- 7431, 18 p.

2. Amani A., Inoussa M.M., Dan Guimbo I., Mahamane A., Saadou M., Lykke5 A.M. (2015). Germination et croissance de quatre espèces de Combretaceae en pépinière. Tropicultura, 2015, 33,2, 135-145.

3. Castell. C. and J. Terradas, (1994). Effects of water and nutrient availability on water relations, gas exchange and growth rate of mature plants and resprouts of Arbutus unedo L. Annals of Botany 73: 595602.

4. Dangbo F. A., Adjonou K., Kokou K., Blaser J. 2019. The socioeconomic contribution of Detarium senegalense seeds to rural livelihoods in Togo (West Africa), International Journal of Biological and Chemical Sciences. 13(3): 1582-1595.

5. Diop N., Ndiaye A., Cisse M., Dieme O., Dornier M., Sock O. 2010. Le ditax (Detarium senegalense J. F. Gmel.) : principales caractéristiques et utilisations au Sénégal, Fruits, vol. 65 (5), 293-306. 
6. Diop N. (2013). Caractérisation du ditax (Detarium senegalense J.F.Gmel) et étude de sa transformation en nectar. Thèse de doctorat Université Cheikh Anta Diop de Dakar, 165 p.

7. Dossa A.K. B., Gouwakinnou N. G., Sourou B. N., Houètchégnon T., Wedjangnon A. A., Odjrado K. B., Ouinsavi C. (2019). Caractérisation structurale des peuplements naturels de Detarium senegalense J.F. Gmel. (Caesalpiniaceae) au Bénin, Afrique de

8. Geny P., Waechter P., Yatchinovsky A. (1992). Environnement et Développement Rural: Guide de Gestion des Ressources Naturelles. Frison-Roche: Paris.

9. Hamawa Y., Baye-Niwah C., Kepwa F. B. F. S., Mapongmetsem P. M., (2020). Effet de Prétraitements sur la Germination des Semences d'Acacia senegal (L.) Willd. (Mimosaceae) dans la Zone Sahélienne du Cameroun. European Scientific Journal, Vol.16, No.3, 12 p.

10. Hamidou A., Dan Guimbo i, Morou B, Taffa S. M., Mahamane, A. (2013). Potential germination and initial growth of Sclerocarya birrea (A. Rich.) Hochst in Niger. Journal of Applied Biosciences 76:64336443.

11. Neuenschwander, P., Sinsin, B. \& Goergen, G. (eds). (2011). Protection de la Nature en Afrique de l'Ouest: Une Liste Rouge pour le Bénin. Nature Conservation in West Africa: Red List for Benin. International Institute of Tropical Agriculture, Ibadan, Nigeria. 365 p.

12. Sabaly K. 2014. Contexte et utilisations locales des fruits de Adansonia digitata et Detarium senegalense au Sine Saloum au Sénégal. Mémoire de Master en Gestion des Ressources Naturelles et Développement Durable au Sénégal, $61 \mathrm{p}$.

13. Yossi H. et al., (2006). Les haies vives au Sahel. État des connaissances et recommandations pour la recherche et le développement. Occasional Paper no. 6. Nairobi : World Agroforestry Centre (ICRAF). 14. https://uses.plantnet-project.org/fr/Detarium_senegalense_(PROTA) (Consulté le 18/10/2018 à 22 heures 35 minutes). 